\title{
Duplication of aortic wall seen by echocardiography
}

\author{
David S. Hirschfeld, ${ }^{1}$ Humberto J. Rodriguez, and Nelson B. Schiller \\ From the Medical Service, San Francisco General Hospital; the Department of Medicine, Veterans Ad- \\ ministration Hospital; and the Department of Medicine, University of California, San Francisco, California, \\ U.S.A.
}

\begin{abstract}
In four patients, echocardiography showed duplication of an aortic wall echo. An aortic dissection was present in only one. Other causes for the echocardiographic pattern were abscess in the interventricular septum, mitral stenosis, and dilatation of the noncoronary sinus of Valsalva. Division of the echo from the mitral ring into two separate posterior aortic wall echoes was seen only in the patient with dissecting aneurysm; it is suggested that this appearance adds to the specificity of the finding of aortic wall duplication by echocardiography in the diagnosis of aortic dissection.
\end{abstract}

The echocardiogram has been shown to be useful in the diagnosis of dissecting aortic aneurysm involving the aortic root (Millward, Robinson, and Craige, 1972; Nanda, Gramiak, and Shah, 1973; Yuste et al., 1974). Findings that suggest dissection are widening or duplication of one or both aortic walls (Millward et al., 1972; Nanda et al., 1973; Yuste et al., 1974) and pronounced variation in aortic root diameter with minor changes in transducer angulation (Nanda et al., 1973). These patterns may, however, be seen in conditions other than aortic dissection (Nanda et al., 1973; Krueger et al., 1975). We are reporting here echocardiograms from four patients which showed apparent duplication of one or both aortic walls. In one patient an aortic dissection was present. In the second there was no aortic dissection, but an abscess in the interventricular septum was found at necropsy. In the third, angiography showed aneurysmal dilatation of the aortic root and noncoronary sinus of Valsalva. In the fourth, reflection from the mitral ring produced an echo parallel with the posterior aortic wall echo.

\section{Methods}

Echocardiograms were performed with a Picker EV 10 echocardiograph with a polaroid film or interfaced with a Honeywell 1856 strip chart recorder and with a Unirad echocardiograph used with a Tektronics strip chart recorder. A focused $2 \cdot 25$ Received 29 September 1975.

1Present address: Valley West Hospital, Saratoga, California, U.S.A.
$\mathrm{MHz} 1.3 \mathrm{~cm}$ transducer was used. Aortic root dimensions were measured from the centre of the anterior aortic wall echo to the centre of the posterior aortic wall echo at end-systole.

\section{Case reports}

\section{Case 1}

This 61-year-old woman had been admitted to hospital in September 1974, with a history of chest pain, shortness of breath, and syncope. A pulmonary arteriogram showed a large filling defect in the right pulmonary artery. She was treated initially with intravenous heparin, and was discharged on treatment with warfarin. In January 1975, a murmur of aortic regurgitation was noted. A chest $x$-ray film showed an increase in heart size.

On admission to San Francisco General Hospital on 11 March 1975, the blood pressure was $130 / 55$ $\mathrm{mmHg}(17 \cdot 3 / 7 \cdot 3 \mathrm{kPa})$ in the left arm and $85 / 60$ $\mathrm{mmHg}(11.3 / 8.0 \mathrm{kPa})$ in the right arm. The pulse was regular at 120 beats $/ \mathrm{min}$. The neck veins were not distended; râles were heard at both lung bases. The first heart sound was quiet; the aortic component of the second sound was accentuated; a third heart sound was present at the apex; there was a grade $3 / 6$ systolic ejection murmur at the base and a grade $2 / 6$ diastolic blowing murmur along the left sternal edge. The liver was not enlarged or tender. There was $2+$ pitting oedema of the ankles. A chest $x$-ray film showed an enlarged cardiac silhouette, a wide mediastinum, and pulmonary venous congestion. The electrocardiogram showed changes of 
anterior wall infarction which had appeared since September 1974. A retrograde aortogram from the right femoral artery showed an aortic dissection, with opacification of both a true and a false lumen and demonstration of a re-entry point in the left external iliac artery. The catheter could not be passed above the lower abdominal aorta. The patient was transferred to the University of California Mcffitt Hospital for operation.
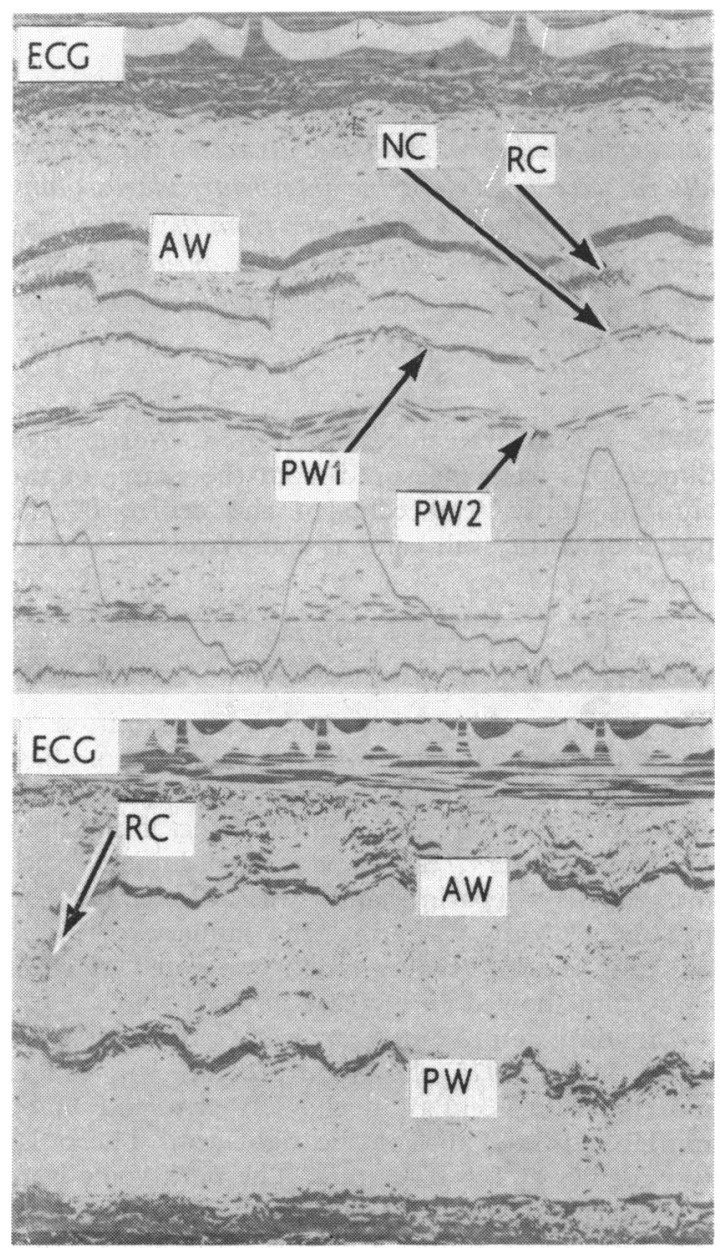

F I G. 1 Echocardiograms in Case 1 before operation. (a) Duplication of posterior aortic wall echo ( $P W 1$ and PW2). Diameter of presumed aortic lumen ( $A W$ to $P W 1$ ) is $25 \mathrm{~mm}$; diameter of the presumed false lumen (PW1 to $P W 2$ ) is $13 \mathrm{~mm}$. (b) With slight cephalad redirection of transducer, aortic diameter increases from 34 to $46 \mathrm{~mm}$. $A W=$ anterior aortic wall; $N C=$ noncoronary cusp; $R C=$ right coronary cusp.
Echocardiograms obtained then showed a thickened anterior aortic wall echo and duplication of the echo from the posterior aortic wall (Fig. 1a). The noncoronary cusp appeared to open only as far as the anterior component of the posterior aortic wall echo. A sweep of the aortic root cephalad from the level of the aortic valve (Fig. 1b) showed a marked increase in the diameter of the aorta with minor change in angulation of the transducer. The distance between the two posterior wall echoes varied with transducer position. In Fig. 1a, a $13 \mathrm{~mm}$ space is present; in Fig. 2a, the space is $7 \mathrm{~mm}$. When the transducer is angled more laterally (Fig. 2b), only a single posterior aortic wall echo is imaged. A sweep from the mitral valve to the aortic root (Fig. 2c) showed the mitral ring echo to be in continuity with both posterior aortic wall echoes.

At operation, an aortic dissection was found to extend into the sinuses of Valsalva. The false lumen was widest over the right and noncoronary cusps. The left coronary artery was sheared off at its origin. Support of the aortic valve was restored by reattaching intima to adventitia at the valve ring. The aorta was replaced with a woven Dacron graft sewn immediately above the valve ring.

Fig. 3 shows an echocardiogram two weeks after operation. Duplication of the posterior aortic wall echo can no longer be seen.

\section{Case 2}

A 35-year-old male user of illicit intravenous drugs was admitted to San Francisco General Hospital on 16 July 1973 with cellulitis on the right arm. No heart murmur was present then. Blood cultures grew Staphylococcus epidermidis. Intravenous administration of cephalothin ( $1 \mathrm{~g}$ every 6 hours) for one week resulted in resolution of the cellulitis; the patient then left the hospital against medical advice.

He was readmitted on 28 August 1973 with a 2-week history of fever, chills, arthralgia, fatigue, and anorexia. The blood pressure was $94 / 40 \mathrm{mmHg}$ $(12.5 / 5.3 \mathrm{kPa})$, the pulse regular at 110 beats/ minute, and the temperature $38.6^{\circ} \mathrm{C}$. The neck veins were not distended. The carotid arterial pulse was collapsing in quality. The lungs were clinically normal. Loud decrescendo diastolic and apical pansystolic murmurs were heard. Petechiae were present on the conjunctivae. Blood cultures grew Serratia marcescens and treatment was started with streptomycin intramuscularly $(1 \mathrm{~g}$ every 12 hours). On the fifth day, the patient was transferred to the University of California Moffitt Hospital for evaluation for aortic valve replacement. Repeat blood cultures were positive for Serratia. On the 


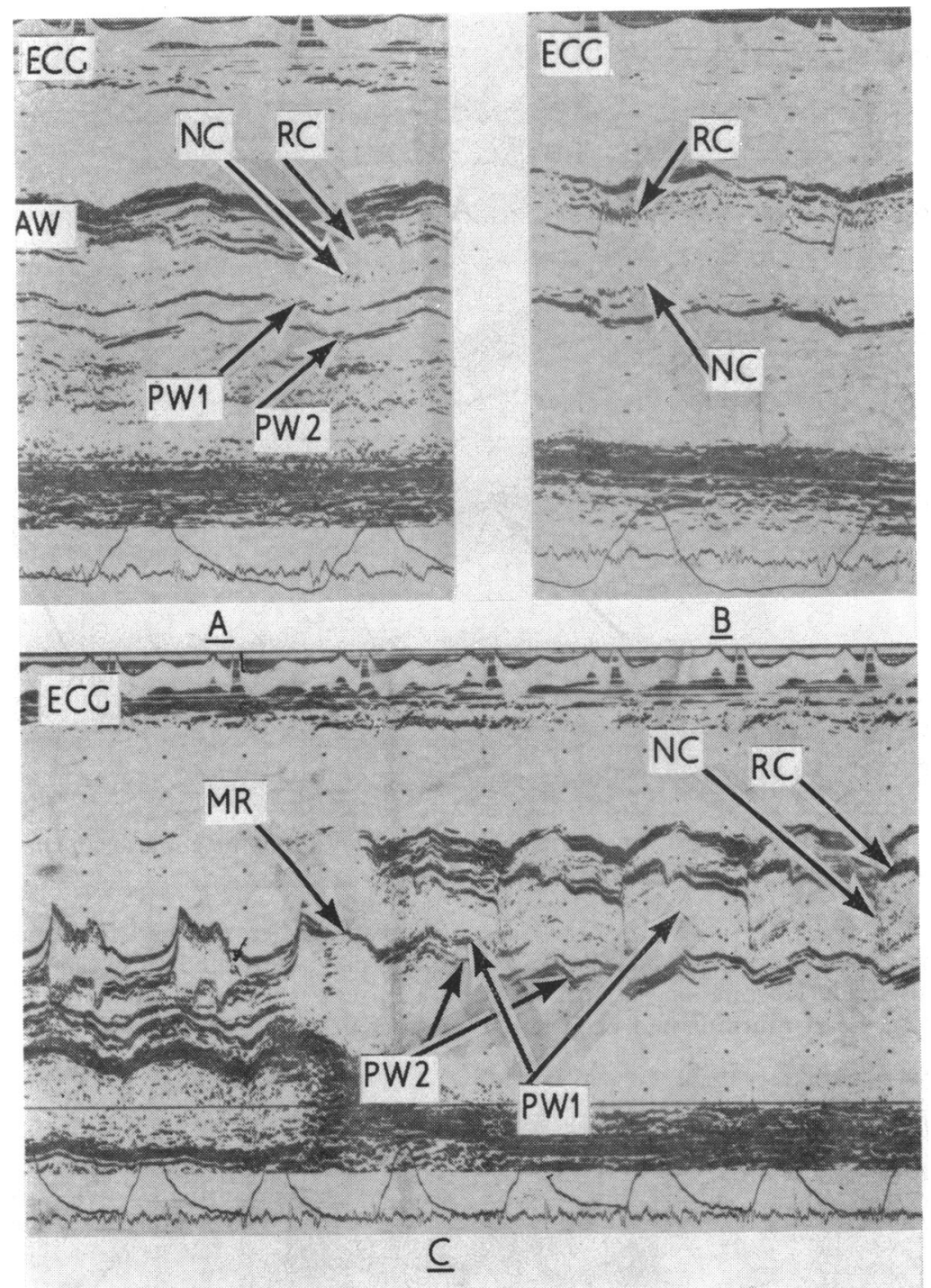

FIG. 2 Echocardiograms in Case 1 before operation. (a) $A 7 \mathrm{~mm}$ space is present between the two posterior aortic wall echoes (PW1 to PW2). (b) With transducer angled more laterally, a single posterior aortic wall echo is seen. The noncoronary aortic cusp is seen in systole to open nearly to the posterior wall. (c) Sweep from mitral valve to aortic root, showing two echoes from posterior aortic wall appearing to arise from echo of mitral ring (MR). See Fig. 1 for abbreviations.

tenth day, râles developed and right bundle-branch block was noted on the electrocardiogram. An echocardiogram (Fig. 4a) showed duplication of the anterior aortic wall echo. Some views showed a cloud of echoes just posterior to the right coronary cusp during systole (Fig. 4b). In addition, prominent vibrations of the anterior mitral leaflet occurred during diastole and the mitral valve closed early. On the 13th day, heart failure worsened, complete heart block developed, and the patient died. No aortic dissection was found at necropsy, but a $30 \mathrm{~mm}$ vegetation was attached to the right coronary cusp, and an abscess was present high in the interventricular septum (Fig. 4b). 


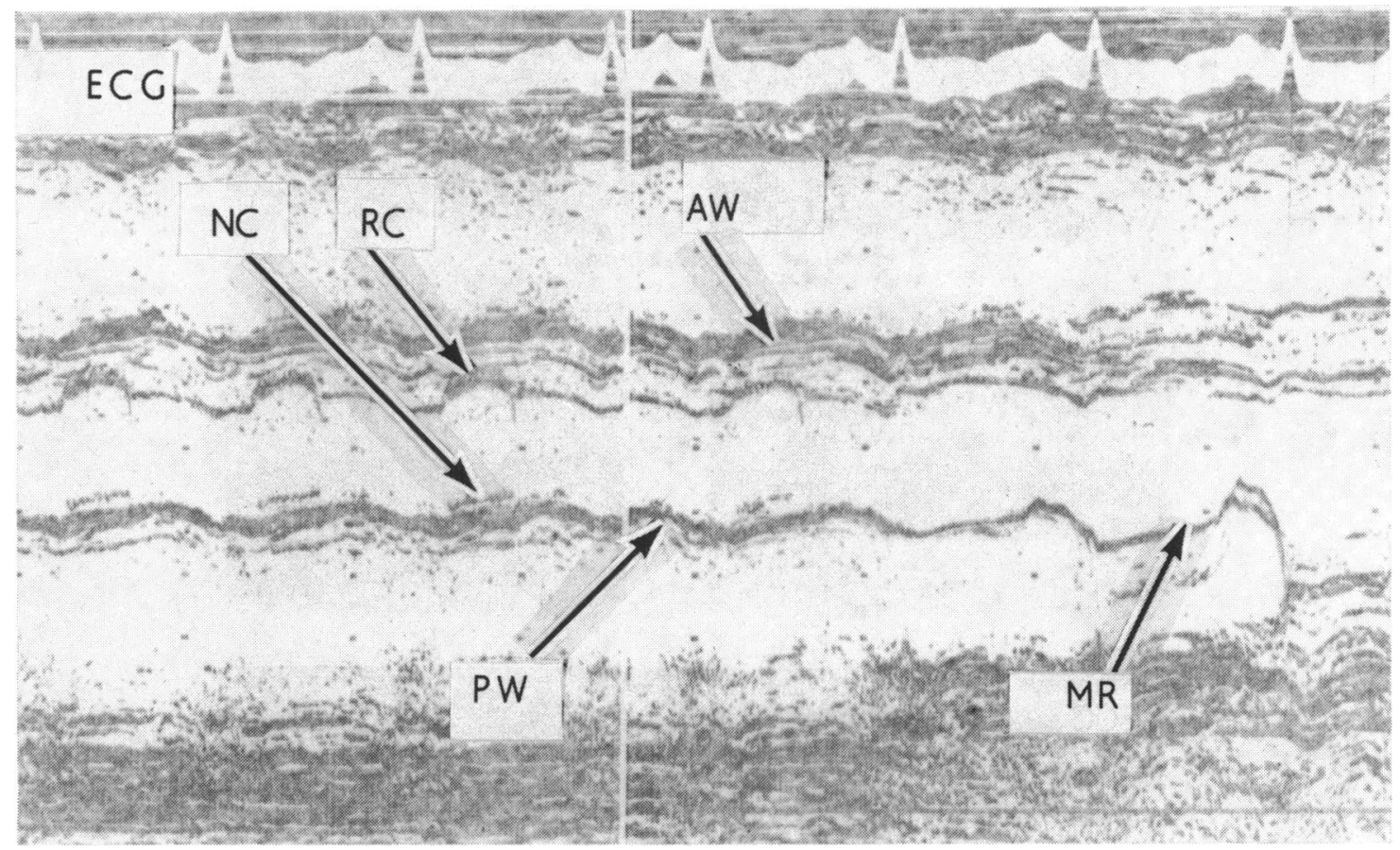

FIG. 3 Echocardiogram in Case 1 after operation. Duplication of posterior aortic wall echo is no longer seen. See Fig. 1 and 2 for abbreviations.

Case 3

A 49-year-old man was admitted to San Francisco Veterans Administration Hospital on 21 April 1975 for investigation. A heart murmur had been noted
25 years earlier. He had had episodic praecordial chest pain, unrelated to exertion, radiating to the back, left shoulder, and neck, accompanied by paraesthesiae in the left hand, with increasing

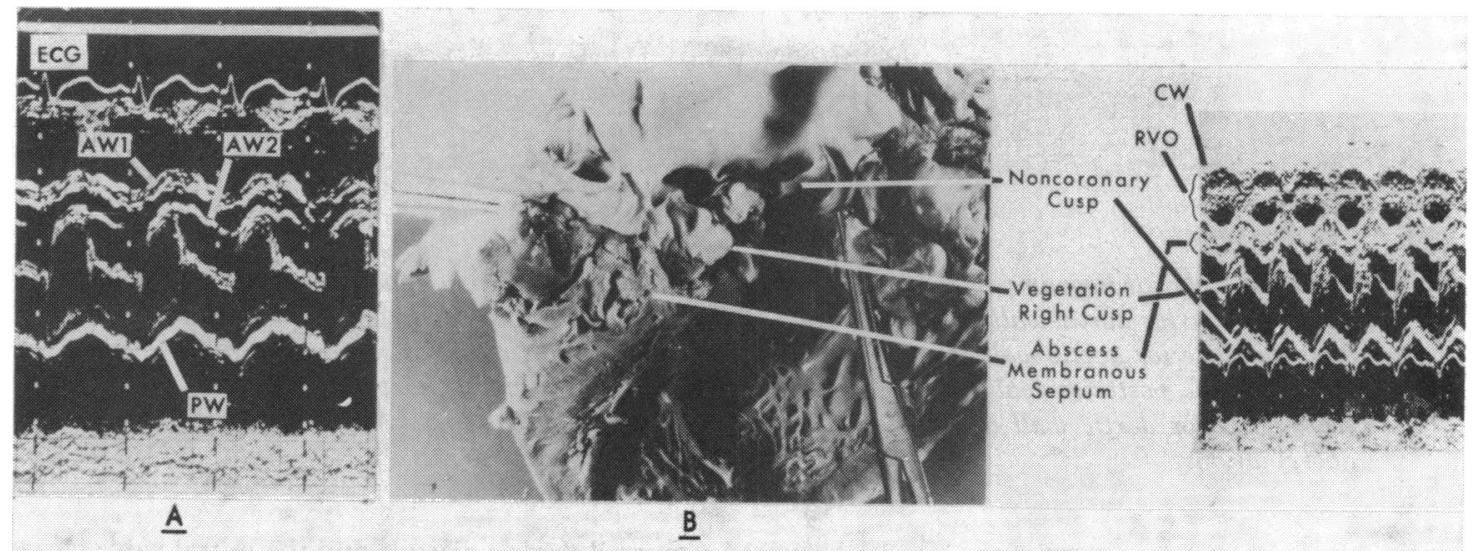

FI G. 4 Echocardiograms in Case 2. (a) Duplication of anterior aortic wall echo; space between $A W 1$ and $A W 2$ is $10 \mathrm{~mm}$. During systole, aortic leaflets open to posterior aortic wall (PW) and to more posterior of the two anterior aortic wall echoes. (b) Necropsy specimen correlated with high gain echocardiogram of aortic root. Mass of fluffy echoes posterior to right coronary cusp during systole is thought to come from vegetation attached to that cusp. CW=chest wall; $R V O=$ right ventricular outflow. 


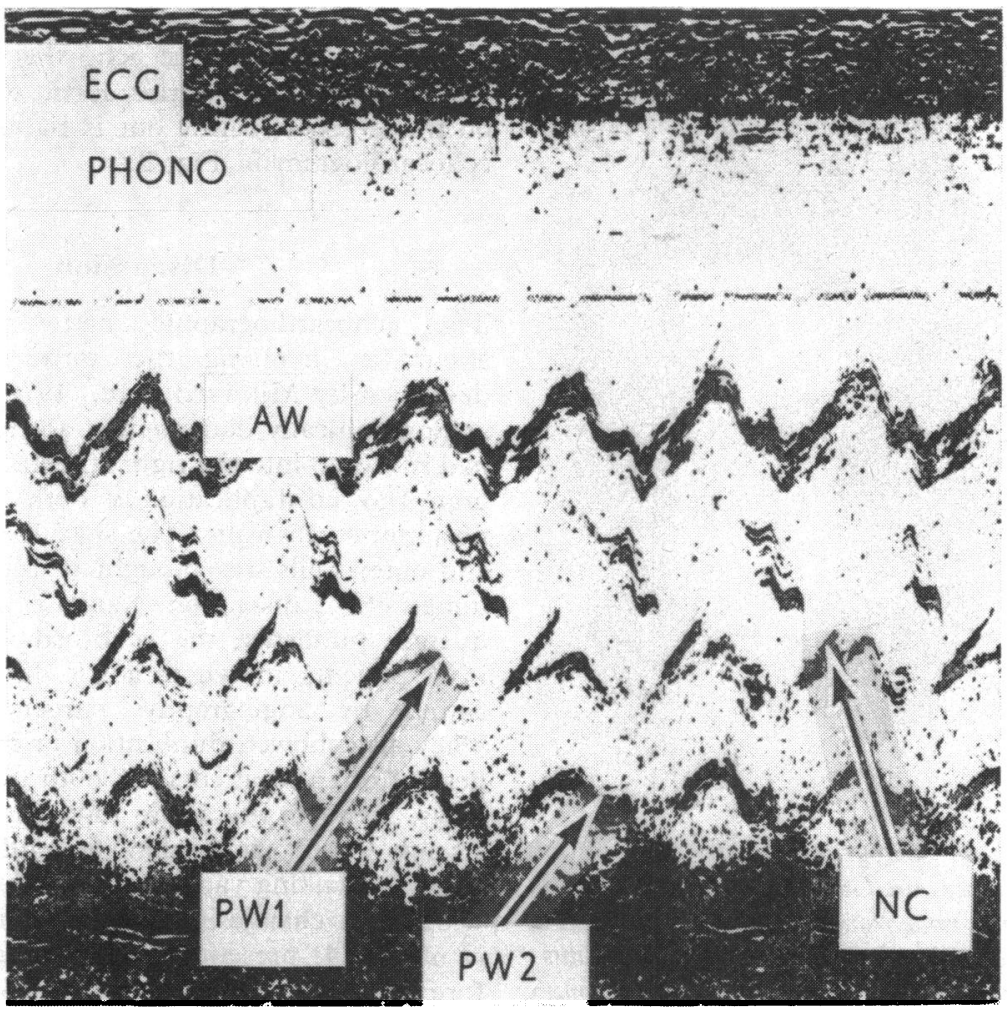

FIG. 5 Echocardiogram of aortic root in Case 3 showing duplication of echo from posterior aortic wall (PW1 and PW2). Aortic valve opens only to more anterior of two posterior aortic wall echoes (PW1). See Fig. 1 for abbreviations.

frequency over the past 20 years.

Blood pressure was $120 / 80 \mathrm{mmHg}(16 \cdot 0 / 10.6$ $\mathrm{kPa}$ ) in both arms and the pulse was regular at 80 beats/min. Carotid pulses were normal. An early systolic click and a grade $1 / 6$ early diastolic blowing murmur were heard along the left sternal edge. Mild pectus excavatum was present; there was no gynaecomastia. The penis was infantile and the right testis was small and soft. There was a normal male escutcheon. VDRL was negative. The electro-

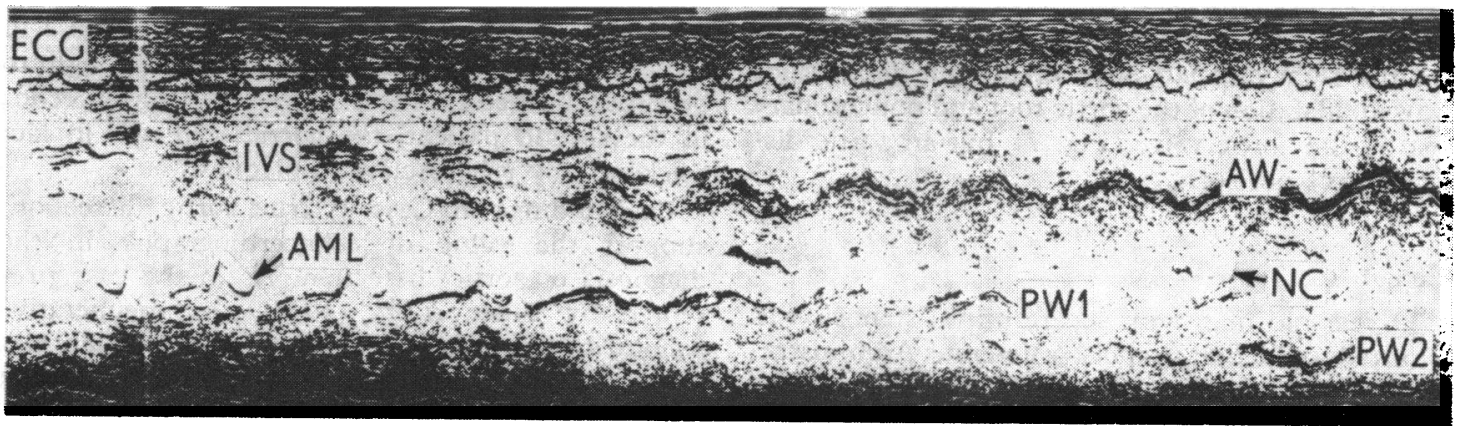

FIG. 6 Echocardiogram in Case 3. Sweep from mitral valve (left) to aortic root (right). Continuity is shown between mitral ring and anterior (PW1) but not posterior (PW2) of two posterior aortic wall echoes. Noncoronary cusp opens only as far as PW1. AML=anterior mitral leaflet; IVS =interventricular septum. 


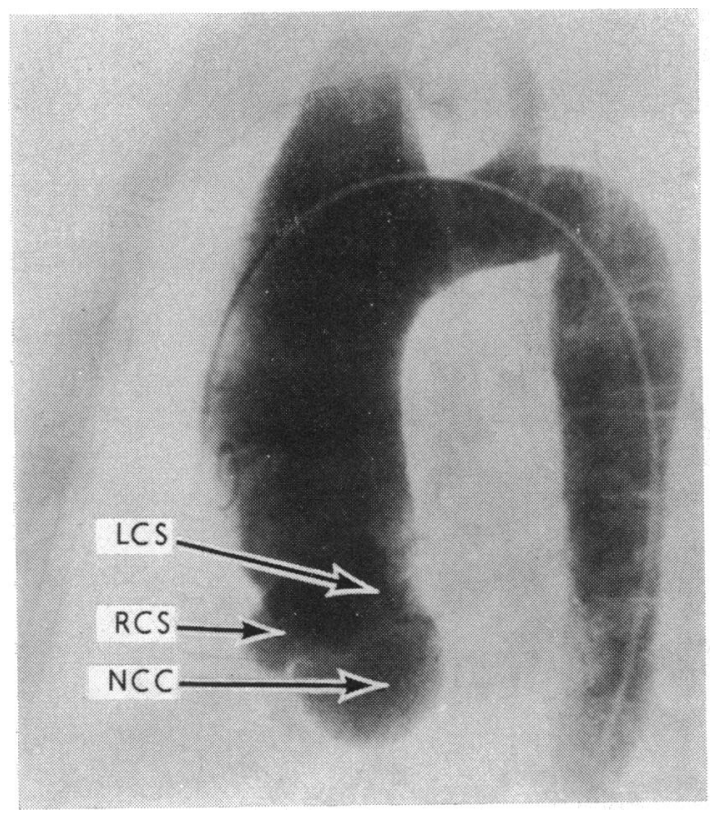

FIG. 7 Frame from aortogram in lateral projection (subtraction technique) in Case 3 showing dilatation of aortic root and noncoronary sinus of Valsalva. No dissection is evident. LCS=left coronary sinus; $R C S=$ right coronary sinus ; $N C C=$ noncoronary cusp.

cardiogram was normal. Chest $x$-ray films suggested dilatation of the aortic root.

An echocardiogram showed an echo moving parallel and $15 \mathrm{~mm}$ posterior to that from the posterior aortic wall (Fig. 5). A sweep cephalad from the mitral ring (Fig. 6) showed continuity between the mitral ring and the more anterior of the two posterior aortic wall echoes. Because of the possibility of a chronic aortic dissection, aortography was performed; aortic root angiograms in the lateral and the right and left anterior oblique projections showed dilatation of the aortic root and noncoronary cusp of the aortic valve (Fig. 7), but an aortic dissection was not seen.

\section{Case 4}

A 39-year-old man with severe mitral stenosis was admitted to the San Francisco Veterans Administration Hospital on 28 March 1975 for insertion of a prosthetic mitral valve. An echocardiogram performed before operation showed apparent duplication of the echo from the posterior aortic wall (Fig. 8). A sweep from the aortic root to the mitral valve (Fig. 9) showed that both apparent posterior aortic wall echoes were in fact reflections from the calcified mitral valve. The echo that probably represented the true posterior aortic wall can be seen on the sweep in Fig. 9 but is barely visible in the echocardiogram in Fig. 8.

\section{Discussion}

The echocardiographic pattern of dissecting aneurysm involving the aortic root was first described by Millward et al. (1972) in a man with angiographically documented aortic dissection that had ruptured into the right atrium. The echocardiogram showed duplication of both the anterior and posterior aortic walls. The space between the inner and outer walls was thought to represent the false lumen of the dissection. Nanda et al. (1973) subsequently published the echocardiographic findings in 6 patients in whom aortic dissection was confirmed by angiography, surgery, or necropsy. They also showed duplication or increase in thickness of one (2 patients) or both (4 patients) aortic walls. In all 6 , the aortic root was widened (42 to $53 \mathrm{~mm}$ outer dimension at end-systole). In 2 patients, striking variation in aortic width was seen with slight change in transducer angulation. Yuste et al. (1974) presented echocardiograms taken before and after repair of a dissecting aneurysm. Duplication of both aortic walls was present before and absent after operation.

Nanda et al. (1973) observed that widening or duplication of the aortic walls could be simulated in calcific aortic valve disease, in pericardial effusion when fluid extends into the transverse sinus, and in patients in whom a catheter has been passed into the pulmonary artery. The possibility of false-positive echocardiographic diagnosis of aortic dissection was also emphasized by Krueger et al. (1975), who described 2 patients with apparent thickening or duplication of the aortic wall. In 1 of these, reflection from the mitral ring was thought to be superimposed on the posterior aortic wall echo; in the second patient, the aortic wall was in fact thickened by atherosclerotic plaques.

Our findings in Case 1 (true aortic dissection) support the value of echocardiography in the diagnosis of aortic dissection. As in the case presented by Yuste et al. (1974), the preoperative echocardiogram showed a false lumen, which was absent in the postoperative study. It is of note that not all views of the aortic root in this patient showed duplication of the posterior aortic wall echo. The positive echocardiographic findings would easily have been missed if multiple sweeps from differing transducer positions had not been per- 

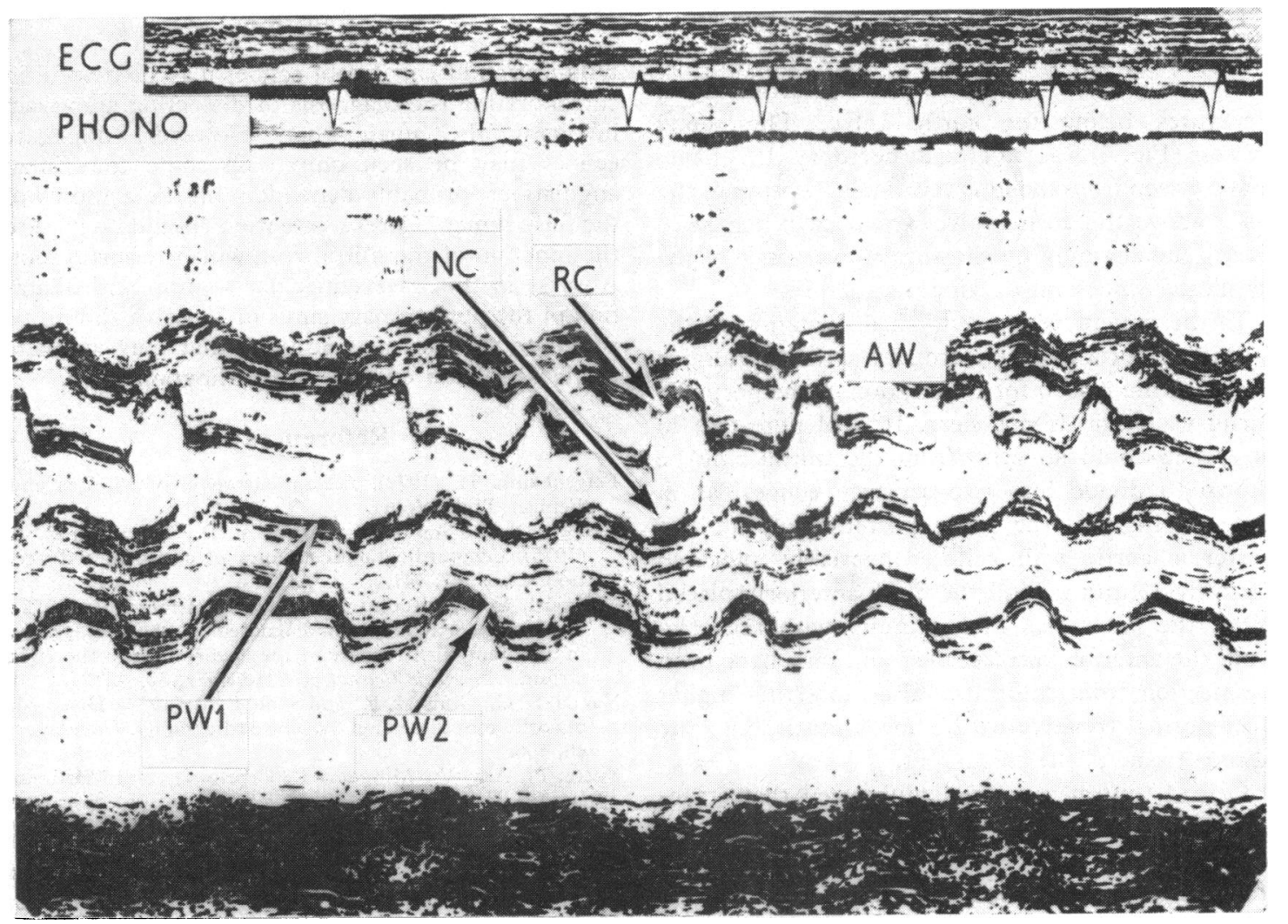

FIG. 8 Echocardiogram in Case 4. Apparent duplication of the echo from posterior aortic wall (PW1 and PW2). See Fig. 1 for abbreviations.

formed. The demonstration that the echo from the mitral ring diverges into two posterior wall echoes (Fig. 2c) is probably valuable in supporting a diagnosis of dissection, as suggested by Krueger et al. (1975). We too would urge careful scanning of the mitral ring-aortic root junction when echocardiography is used to corroborate a suspected aortic dissection.

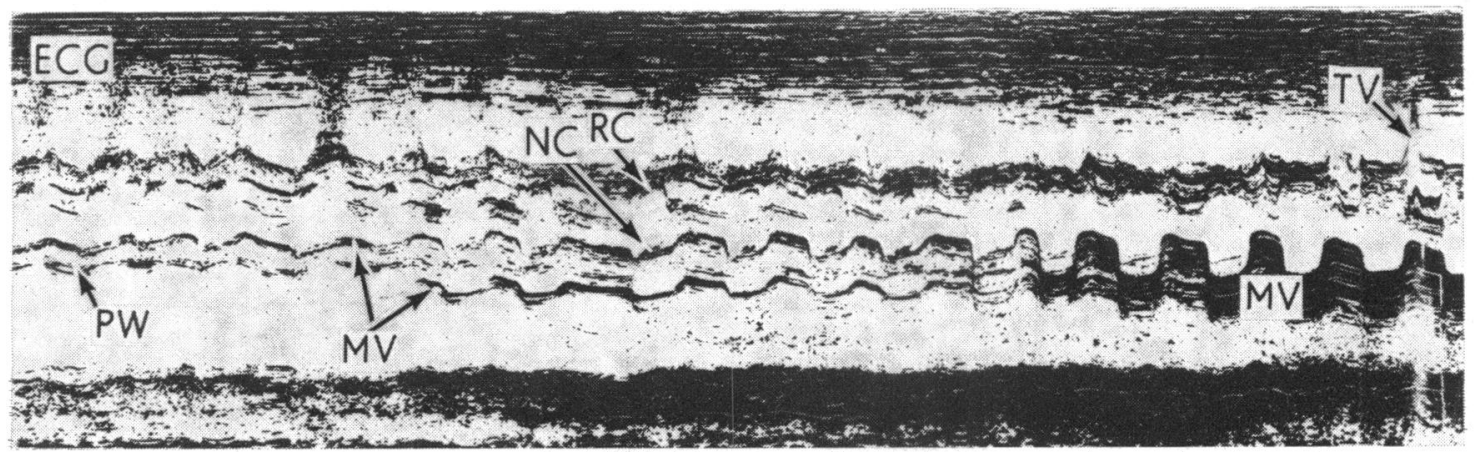

FIG. 9 Echocardiogram in Case 4. Sweep from aortic root to mitral valve (MV). Dense echoes from calcified mitral valve are present at level of aortic valve and simulate duplication of posterior aortic wall echo. Echo that probably represents true posterior aortic wall (PW) is barely visible in middle of sweep. TV=tricuspid valve; see Fig. 1 for abbreviations. 
Case 2 (abscess in the interventricular septum) is of interest because the apparent double echo of the anterior aortic wall may have arisen from structures below the aortic valve. The septal abscess (Fig. 4) was bulging at necropsy. It is likely that a sweep from the interventricular septum to the aorta above the aortic valve would have helped to clarify the anatomy by showing convergence of the duplicate echoes into a single structure.

In the patient with aneurysmal dilatation of the ascending aorta and noncoronary sinus of Valsalva (Case 3), the reason for duplication of the posterior aortic wall echo is not clear. It is of note that in no sweep could an echo from the mitral ring be shown to divide into two separate echoes, as in Case 1. Instead, the more posterior of the two posterior aortic wall echoes invariably appeared abruptly $15 \mathrm{~mm}$ behind the more anteriorly placed echo; the latter was in continuity with the echo from the anterior mitral leaflet, and may have been a reflection from the mitral ring, inserting higher than normal (relative to the aortic cusps) into the fibrous tissue of the aortic root.

Case 4 (mitral stenosis) emphasized that structures adjacent to one another anatomically may appear superimposed in the echocardiogram (Feigenbaum, 1972), because of the width of the echo beam.

\section{Conclusions}

Duplication of aortic root echoes may be helpful in corroborating the diagnosis of dissecting aneurysm involving the aortic root. However, duplicate echoes may be seen only with some transducer angulations, probably dependent on the anatomy of the false lumen. Careful attention should be paid to the junction of the mitral ring with the aortic root. Abscess in the interventricular septum and dilatation of the noncoronary sinus of Valsalva should be added to the list of conditions that may simulate aortic dissection on the echocardiogram.

\section{References}

Feigenbaum, H. (1972). Echocardiography, p. 15. Lea and Febiger, Philadelphia.

Krueger, S. K., Starke, H., Forker, A. D., and Eliot, R. S. (1975). Echocardiographic mimics of aortic root dissection. Chest, 67, 441.

Millward, D. K., Robinson, N. J., and Craige, E. (1972). Dissecting aortic aneurysm diagnosed by echocardiography in a patient with rupture of the aneurysm into the right atrium. American fournal of Cardiology, 30, 427.

Nanda, N. C., Gramiak, R., and Shah, P. M. (1973). Diagnosis of aortic root dissection by echocardiography. Circulation, 48, 506.

Yuste, P., Aza, V., Minguez, I., Cerezo, L., and MartinezBordiu, C. (1974). Dissecting aortic aneurysm diagnosed by echocardiography. British Heart fournal, 36, 111 .

Requests for reprints to Editorial Office, San Francisco General Hospital, 1001 Potrero Avenue, San Francisco, California 94110, U.S.A. 